\title{
All quiet on the Western front? New evidence for massive star formation in Sgr C
}

\author{
Sarah Kendrew ${ }^{1,2} \dagger$, Adam Ginsburg ${ }^{3}$, Katharine Johnston ${ }^{1}$, \\ Henrik Beuther ${ }^{1}$, John Bally ${ }^{3}$, Claudia J. Cyganowski ${ }^{4}$ and \\ Cara Battersby ${ }^{3}$ \\ ${ }^{1}$ Max-Planck-Institut für Astronomie, Königstuhl 17, 69117 Heidelberg, Germany \\ ${ }^{2}$ Sub-Dept of Astrophysics, University of Oxford, Keble Road, Oxford OX1 3RH, \\ United Kingdom \\ ${ }^{3}$ CASA, University of Colorado at Boulder, UCB 389, Boulder, CO 80309, USA \\ ${ }^{4}$ Harvard-Smithsonian Center for Astrophysics, Cambridge, MA 02138, USA
}

\begin{abstract}
We summarize here our recent findings from near-infrared spectroscopy and $1 \mathrm{~mm}$ line and continuum observations of a recently identified extended green object (EGO) in Sgr C, whose observational characteristics suggest early-stage massive star formation is taking place. Located on the outskirts of the massive evolved Hil region associated with $\mathrm{Sgr} \mathrm{C}$ in the Western central molecular zone (CMZ), the EGO measures $\sim 10^{\prime \prime}(0.4 \mathrm{pc}$ at $8.5 \mathrm{kpc})$. We confirm that early-stage star formation is taking place on the periphery of the $\mathrm{Sgr}$ C HII region. The data show clear detections of two protostellar cores and several knots of $\mathrm{H}_{2}$ and Brackett $\gamma$ emission alongside a previously detected compact radio source. We calculate the cores' joint mass to be $\sim 10^{3} \mathrm{M}_{\odot}$, with column densities of $1-2 \times 10^{24} \mathrm{~cm}^{-2}$. The host molecular clouds mass is approximately $10^{5} \mathrm{M}_{\odot}$. Despite these favorable conditions, the cloud is curiously devoid of any further star formation, making it comparable to other remarkably quiescent clouds, such as G0.253 in the Eastern CMZ.
\end{abstract}

\section{Introduction: Sgr C and EGO G359.44-0.102}

$\mathrm{Sgr} \mathrm{C}$ is the only known star forming region in the Western central molecular zone (CMZ). Its main characteristics are a 10-pc HiI region, a distinctive non-thermal filament and a molecular cloud, measuring $\sim 16 \times 9 \mathrm{pc}$ in size and thought to harbor $\leqslant$ $10^{5} \mathrm{M}_{\odot}$ (Lang et al. 2010; Liszt \& Spiker 1995; Lis et al. 1991).

The first evidence of high mass star formation in the Sgr C cloud was reported by Forster \& Caswell (2000), who detected a faint $8-9 \mathrm{GHz}$ radio source measuring $0.06 \times 0.01 \mathrm{pc}$ near its tip. Using data from the Spitzer GALCEN survey (Stolovy et al. 2006), YusefZadeh et al. (2009) identify a region of extended $4.5 \mu \mathrm{m}$ emission - a so-called extended green object (EGO; Cyganowski et al. (2008)) - within $\sim 5^{\prime \prime}$ from the radio source. Numerous studies have found EGOs to be strongly associated with early-stage high mass star formation and outflows (De Buizer \& Vacca 2010; Cyganowski et al. 2009). Three $\mathrm{CH}_{3} \mathrm{OH}$ masers are seen within the EGO at velocities consistent with the $\mathrm{Sgr} \mathrm{C}$ systemic velocity ( -55 to $-65 \mathrm{~km} \mathrm{~s}^{-1}$ ), supporting this scenario (Chambers et al. 2011).

The new data shown here confirm that massive star formation is taking place in the massive Sgr C molecular cloud. We detect the presence of two protostars driving at least one outflow, alongside the previously detected radio source.

$\dagger$ Present address: Sub-Dept of Astrophysics, University of Oxford, Keble Road, Oxford OX1 3RH, United Kingdom. email: sarah.kendrew@astro.ox.ac.uk 

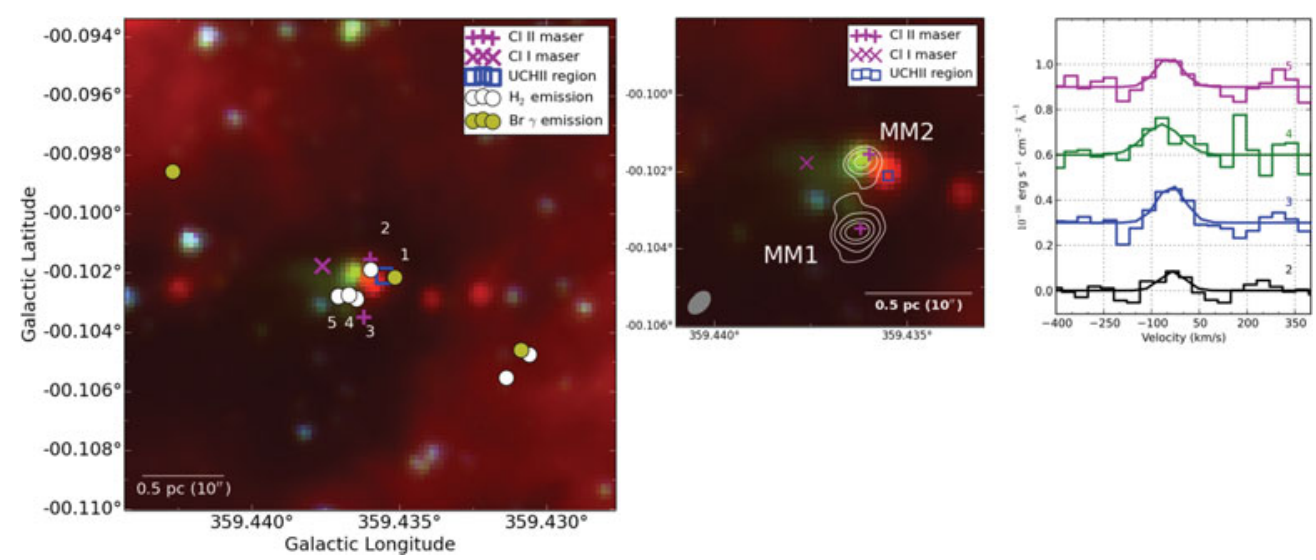

Figure 1. New observational data for the EGO G359.44-0.102. Left: 3.6/4.5/8.0 $\mu \mathrm{m}$ Spitzer image of G359.44-0.102, indicating the location of $\mathrm{CH}_{3} \mathrm{OH}$ masers, the radio source and the IR emission knots. Symbols as indicated in the legend. Middle: as left, with the SMA 280 $\mathrm{GHz}$ continuum contours showing 2 protostellar sources. Right: $\mathrm{H}_{2}$ spectra at $2.12 \mu \mathrm{m}$ for the numbered knots. [A COLOR VERsion IS AVAILABLE ONLINE.]

\section{Observations and results}

We obtained $K$-band spectroscopy with the TripleSpec spectrograph on the 3.5 -m ARC telescope at Apache Point Observatory, using 4 different slit positions covering the EGO. We detected $\mathrm{H}_{2}$ emission at $2.12 \mu \mathrm{m}$ at 4 locations towards $4.5 \mu \mathrm{m}$ emission (Figure 1), consistent with the possible presence of an outflow.

At millimeter wavelengths, $280 \mathrm{GHz}$ continuum data from the Submillimeter Array (SMA; P. I. Kauffmann) show the presence of two protostellar sources towards the EGO (Figure 1). Using integrated flux measurements and assuming a distance of $8.5 \mathrm{kpc}$, temperature of 20K, and a dust-to-gas ratio of 76 (Draine 2011), we estimate their masses to be approximate $668 \mathrm{M}_{\odot}$ for MM1 and $380 \mathrm{M}_{\odot}$ for MM2 with an uncertainty of a factor 2-4. Peak column densities are $1-2 \times 10^{24} \mathrm{~cm}^{-2}$ for both sources.

The observational data presented here convincingly demonstrate that early-stage massive star formation is taking place in the $\mathrm{Sgr} \mathrm{C}$ cloud at 3 sites, likely to be driving at least one outflow.

\section{No further star formation in the Sgr C cloud}

Using $870 \mu \mathrm{m}$ continuum data from the ATLASGAL survey (Schuller et al. 2009; Contreras et al. 2013) we calculate the total mass of the Sgr C molecular cloud to be $\sim 10^{5} \mathrm{M}_{\odot}$, consistent with previous estimates (Lis et al. 1991). Source averaged column densities are in the range $1.5-3.5 \times 10^{22} \mathrm{~cm}^{-2}$. Despite these conditions we find no further tracers of star formation outside of G359.44-0.102. As such, the cloud shares similarities with G0.253 ("the Brick") and its massive quiescent fellow clouds ("Bricklets") on the Eastern side of the CMZ (Lis \& Carlstrom 1994; Longmore et al. 2012; Kauffmann et al. 2013; Immer et al. 2012).

\section{References}

Chambers, E. T., Yusef-Zadeh, F., \& Roberts, D. 2011, ApJ 733, 42

Contreras, Y., et al. 2013, A\& $A$ 549, A45

Cyganowski, C. J., et al. 2008, AJ 136, 2391 
Cyganowski, C. J., Brogan, C. L., Hunter, T. R., \& Churchwell, E. 2009, ApJ 702, 1615

De Buizer, J. \& Vacca, W. 2010, AJ 140, 196

Draine, B. T. 2011, Physics of the Interstellar and Intergalactic Medium

Forster, J. R. \& Caswell, J. L. 2000, ApJ 530, 371

Immer, K., Menten, K. M., Schuller, F., \& Lis, D. C. 2012, A\&A 548, A120

Kauffmann, J., Pillai, T., \& Zhang, Q. 2013, arXiv, preprint, arXiv:1301.1338

Lang, C. C., Goss, W. M., Cyganowski, C., \& Clubb, K. I. 2010, ApJS 191, 275

Lis, D., Carlstrom, J., \& Keene, J. 1991, ApJ 380, 429

Lis, D. C. \& Carlstrom, J. E. 1994, ApJ 424, 189

Lis, D. C., Menten, K. M., Serabyn, E., \& Zylka, R. 1994, ApJ Lett. 423, L39

Liszt, H. \& Spiker, R. 1995, ApJS 98, 259

Longmore, S. N., et al. 2012, ApJ 746, 117

Schuller, F., et al. 2009, A\&A 504, 415

Stolovy, S., et al. 2006, J. Phys. Conf. Ser. 54, 176

Yusef-Zadeh, F., et al. 2009, ApJ 702, 178 